\title{
ARE ELFS LITTLE MONSTERS?
}

\author{
R.P.NORRIS \\ Division of Radiophysics, CSIRO \\ PO Box 76, \\ Epping, NSW 2121 \\ Australia
}

\begin{abstract}
High resolution radio observations of Seyferts, starbursts, and ELFs are presented which suggest that (i) starburst galaxies do not generally possess a Seyfert-like core, (ii) some ELFs do have a Seyfert core, but only those with a Seyfert-like spectrum, (iii) other ELFs are more likely to be powered by starburst activity. Double compact sources are present in several ELFs, suggesting that we might be seeing the nuclei of two merging galaxies, and that the ELFs are conceived by the coupling of two monsters.
\end{abstract}

\section{Introduction}

The origin of the enormous total luminosity of ELFs (extremely luminous far-infrared galaxies) is the subject of considerable debate, and both Seyfert and starburst nuclei have been suggested. Radio observations offer a means to distinguish between these models, and so data from the $275-\mathrm{km}$ Parkes-Tidbinbilla Interferometer (PTI) are presented here. The PTI has a lobe spacing of $\sim 0.1 \mathrm{arcsec}$ at $2.3 \mathrm{GHz}$, corresponding to a size of 10 to $200 \mathrm{pc}$ for the redshift range ( 0.01 to 0.2$)$ of the sources dicussed here. The PTI sensitivity limit of $5 \mathrm{mJy}$ means that it is sensitive only to brightness temperatures in excess of $10^{5} \mathrm{~K}$. Since starburst activity generates radio emission with a typical brightness temperature of about $10^{4} \mathrm{~K}$, the PTI is a well-matched filter to discriminate between starburst and Seyfert activity.

\section{Seyferts and starbursts}

It is now established that a significant fraction of the energy of Seyfert galaxies may be provided by starburst activity, and that at least some starburst galaxies have compact cores. This therefore raises the question of whether there is any real difference between Seyfert and starburst galaxies. For example, could the apparent difference be an orientation effect, with starburst galaxies simply being Seyfert galaxies with obscured cores?

Our PTI observations, shown in the first two columns of Fig. 1, suggest that this is not the case. Of a mixed bag of Seyferts and starbursts chosen from the literature with a similar redshift distribution, it is clear that the Seyferts have a much higher incidence of detectable pcsized cores. Details of some of these observations are given by Norris et al. (1988b).

Observations on a more carefully selected sample are continuing to establish the statistical 
significance of this result.

\section{ELFs and monsters}

A number of ELFs were selected from a large sample of cool, optically-faint infrared sources being studied in a related program. An optical spectrum of each has been obtained on the Anglo-Australian Telescope, allowing their classification into those with and without Seyfertlike spectra. The results of PTI observations of these are shown in the second two columns of Fig. 1. About one third of the ELFs with Seyfert spectra were detected, whereas none of those without Seyfert spectra were detected. We conclude that obscured quasar nuclei such as that in Arp 220 are rare amongst ELFs, except when they betray themselves by a Seyfert-like spectrum. VLA maps of Arp 220 (Norris, 1988) and other ELFs (Norris et al., 1988a) show that several of these have double nuclei, suggesting that they are triggered by mergers.

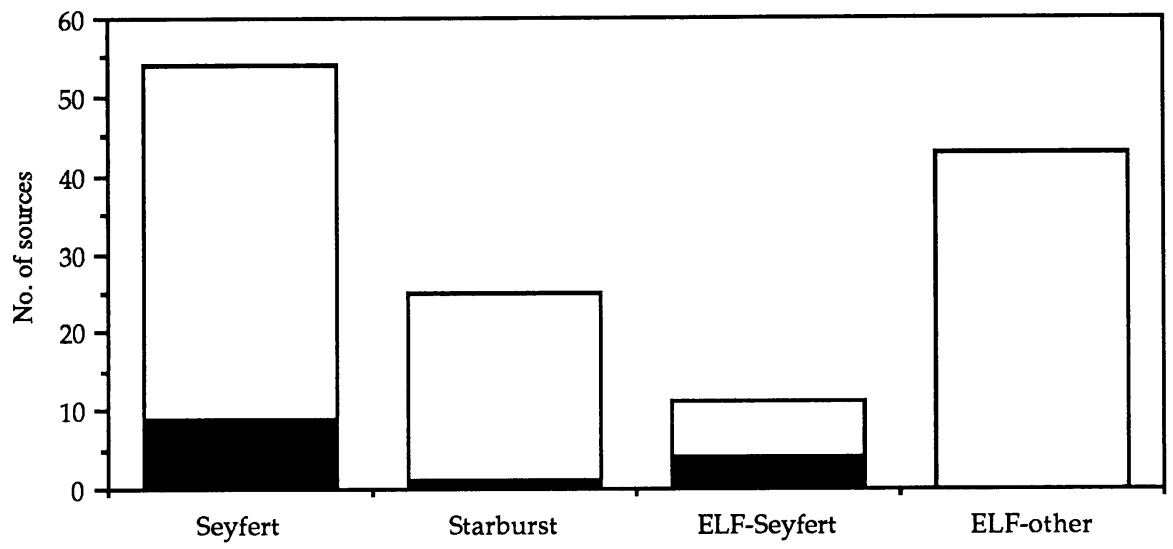

Fig. 1. Results of PTI observations, which are sensitive only to bright compact cores. Each open column shows the number of sources observed in each category, with the filled areas representing the number of sources detected.

\section{Acknowledgement}

I thank my colleagues David Allen, Mike Kesteven, Dick Sramek, and Euan Troupe for permission to present data prior to publication elsewhere.

\section{References}

R.P. Norris, 1988, Mon. Not. R. astr. Soc, 230, 345.

R.P. Norris, D.A. Allen, and P.F. Roche, 1988a, Mon. Not. R. astr. Soc., in press.

R.P. Norris, M.J. Kesteven, D.A. Allen, and E. Troupe, 1988b, Mon. Not. R. astr. Soc., in press. 


\section{DISCUSSION}

ULRICH Arp 220 has been discussed many times in recent years. It offers the well known dilemma that its IR spectrum does not look like that of a heavily reddened typical quasar (like $3 C$ 273), but on the other hand if the infrared radiation is due to stars, then the $6 \mathrm{~cm}$ flux is too faint as compared to the flux expected from a normal star forming region. Do your observations help to resolve this puzzle?

NORRIS It would be rash to say that the dilemma has been solved, but the problem isn't looking quite so intractable. There is clearly a lot of starburst activity in Arp 220 which dominates the optical and near-IR spectrum. The far IR spectrum, however, is dominated by a lot $\left(10^{10} M_{\odot}\right)$ of cool dust in the inner kpc, and this seems to be the dominant total luminosity mechanism in Arp 220. This dust is presumably heated by the quasar nuicleus. We have to be a bit careful when comparing it to $3 C 273$ in view of the enormous extinction $\left(A_{v}>40 \mathrm{mag}\right)$ to the center, so we shouldn't expect the IR spectra to be too similar.

BIERMANN $5 \mathrm{GHz}, 1.3 \mathrm{~mm}, 60 \mu \mathrm{m}$ and $2 \mathrm{keV}$ observations combined clearly demonstrate a difference between starbursts and monsters: in a recent paper (Chini, Kreysa, Biermann, 1988 Astron. Astrophys, submitted) we show that, e.g. optically selected quasars have far infrared to $\mathrm{mm}, \mathrm{X}$-ray spectra showing that they are extended dusty regions powered by a monster.

NORRIS That's an interesting result. It looks like this particular question at least has now been answered. 